\title{
A Transfer of Training Study of Control Loader Dynamics
}

\author{
Frank M. Cardullo ${ }^{1}$, Anthony A. Stanco ${ }^{2}$ \\ Man-Machine Systems Laboratory, Department of Mechanical Engineering, SUNY Binghamton, Binghamton, NY, \\ 13902-6000 \\ Lon Kelly ${ }^{3}$ \\ Unisys Corporation, Hampton, VA, 23666 \\ and \\ Jacob Houck ${ }^{4}$, Richard Grube ${ }^{5}$ \\ NASA LaRC, Hampton, VA, 23665
}

\begin{abstract}
The control inceptor used in a simulated vehicle is an important part in maintaining the fidelity of a simulation. The force feedback provided by the control inceptor gives the operator important cues to maintain adequate performance. The dynamics of a control inceptor are typically based on a second order spring mass damper system with damping, force gradient, breakout force, and natural frequency parameters. Changing these parameters can have a great effect on pilot or driver control of the vehicle. The neuromuscular system has a very important role in manipulating the control inceptor within a vehicle.

Many studies by McRuer, Aponso, and Hess have dealt with modeling the neuromuscular system and quantifying the effects of a high fidelity control loader as compared to a low fidelity control loader. Humans are adaptive in nature and their control behavior changes based on different control loader dynamics. Humans will change their control behavior to maintain tracking bandwidth and minimize tracking error.

This paper reports on a quasi-transfer of training experiment which was performed at the NASA Langley Research Center. The quasi transfer of training study used a high fidelity control loader and a low fidelity control loader. Subjects trained in both simulations and then were transferred to the high fidelity control loader simulation. The parameters for the high fidelity control loader were determined from the literature. The low fidelity control loader parameters were found through testing of a simple computer joystick.

A disturbance compensatory task is employed. The compensatory task involves implementing a simple horizon out the window display. A disturbance consisting of a sum of sines is used. The task consists of the subject compensating for the disturbance on the roll angle of the aircraft. The vehicle dynamics are represented as $1 / \mathrm{s}$ and $1 / \mathrm{s}^{2}$. The subject will try to maintain level flight throughout the experiment. The subjects consist of non-pilots to remove any effects of pilot experience.

First, this paper discusses the implementation of the disturbance compensation task. Second, the high and low fidelity parameters used within the experiment are presented. Finally, an explanation of results from the experiments is presented.
\end{abstract}

${ }^{1}$ Professor, Department of Mechanical Engineering, Associate Fellow AIAA

${ }^{2}$ Graduate Student, Department of Mechanical Engineering, Student Member

3 Software Engineer, Simulation Development and Analysis Branch

${ }^{4}$ Distinguished Research Associate, Simulation Development and Analysis Branch, Associate Fellow AIAA

${ }^{5}$ Electrical Engineer, Simulation Development and Analysis Branch 


\section{Introduction}

The design of flight training simulators requires that the pilot is presented with cues of the dynamic environment to the extent necessary to learn the task being trained. If the pilot is able to transfer the skills he or she has learned in the simulator to the aircraft then it is said that the training transfer is high. This idea of training transfer has been difficult to quantify. Very few studies have been done to measure training transfer between the simulator and the actual aircraft. A major reason for this is that full transfer of training (TOT) studies are difficult to do because they require many hours of flight time and are consequently very expensive. The concept of using quasitransfer of training studies has emerged over the years to mitigate the aforementioned difficulty. A Quasi-TOT study is one in which the pilot trains in one simulator environment and transfers to another simulator environment. There is dispute among researchers as to whether this paradigm is in fact predictive of the actual transfer of training. This paradigm has been used extensively to attempt to justify the need for motion cueing devices in flight simulators. This paper reports on a Quasi-TOT study attempting to evaluate the training effect of a high fidelity vs. low fidelity control loading system. The control loading system was chosen as the simulator subsystem for this study because it is the primary control interface with the simulation and presents a tight control loop to the operator.

The hypothesis advanced by one of the authors was that there would be little difference in the TOT between the two levels of fidelity of the control loading. In other words the outcome would illustrate little value in the use of the high fidelity control loader in training, at least for the task chosen. This had been the case with several motion/no motion Quasi-TOT studies. A simple roll axis disturbance nullification task was chosen for the study. Twenty subjects, ten in each group were selected from the general population at the lab. No flying skills were necessary for the task. The subjects were exposed to two sets of dynamics, $1 / \mathrm{s}$ and $1 / \mathrm{s}^{2}$. At the time of submission the data collection was continuing and only about half of the subjects were included in the results presented, although, there were an equal number from each group.

The main objectives of these experiments are enumerated as follows. First the literature was consulted in order to obtain the high fidelity control loader parameters. The low fidelity characteristics were obtained from tests in our laboratory using a game controller joystick. Next, both sets of parameters were implemented in the simulator. The Quasi-TOT was designed along with the disturbance task. Finally, the subjects were run and data collected and analyzed. The real-time quasi-transfer of training experiments, which were conducted at NASA Langley Research Center, on the Generic Flight Deck (GFD), provided the necessary data for analysis. The use objective metrics, and analyses of subject performance is discussed. Also, any future recommendations will be discussed to improve the control loader experimentation.

\section{Background}

The vehicle simulation design process relies upon principles of human perception to present task appropriate cues to the trainee. Visual, vestibular, and proprioceptive feedback are the main components of human perception used for vehicle control. These components work together with the cognitive system to stimulate a characteristic human control strategy. The fidelity of the simulation depends on how closely the performance of the simulator matches the vehicle. The purpose of simulation is to maintain high fidelity while keeping within the constraints of the simulator. The simulator has motion limitations that make it difficult to recreate the same accelerations and velocities experienced in the vehicle. This section discusses background on aircraft and flight simulation control systems, the neuromuscular system, and transfer of training studies.

\section{A. Aircraft Control System Dynamics}

Many new aircraft have moved to mechanical hydraulically boosted and fly-by-wire force feel systems which have unknown effects on pilot performance. The fly-by-wire and mechanical hydraulically boosted systems do not have any direct physical connection to the control surfaces of the aircraft. Both a mechanical hydraulically boosted and fly-by-wire control system use hydraulics to reduce the forces required to move the control surfaces. Mechanical hydraulically boosted and fly-by-wire systems utilize an artificial force feel device to create the force feedback cues that arrive from the aerodynamic forces on the control surfaces. In the same manner, simulators use a control loader system to simulate the control inceptor dynamics in the real world vehicle. Within this section, there is a discussion on the background of aircraft and simulator fight control systems.

The flight control system in aircraft before WWI consisted of direct mechanical linkages to the control surfaces (Takats, 2011). This type of flight control system is referred to as a reversible flight control system whereby the aerodynamic forces on the control surfaces are felt directly by the pilot. An example of a direct mechanical flight control system is shown below in Figure 1 a). 


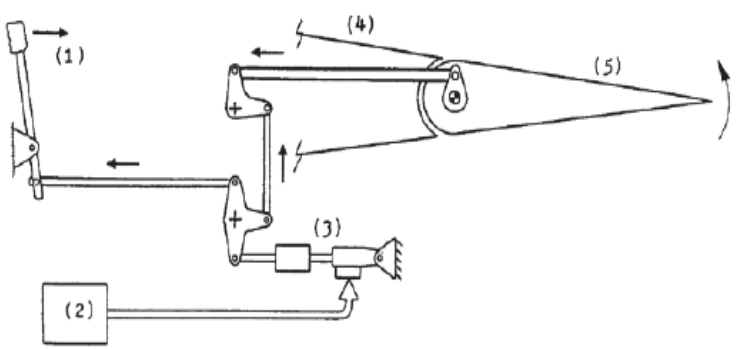

Figure 1 a) Direct Mechanical Flight Control System (Takats, 2011)

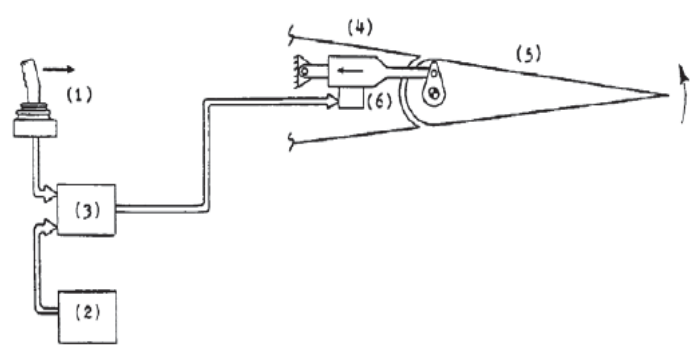

b) Fly-By-Wire Flight Control System (Takats, 2011)

In Figure $1 \mathrm{a}$ ), the input force by the operator causes the control inceptor (1) to deflect which transfers to the control surface (5) through a series of direct linkages and hinges. This causes an increased aerodynamic force on the control surface (5) which creates a hinge moment that create force feedback directed back to the control inceptor via the linkages and hinges. The gearing of the linkages provides a certain amount of mechanical advantage but is limited by the space available within aircraft. Also shown in Figure $1 \mathrm{a}$ ) is the autopilot (2) system, which applies a signal to a servo valve to deflect the control surface (5).

On larger aircraft, hydraulically boosted flight control systems are typically used. Two types of hydraulically boosted flight control systems exist: mechanical and fly-by-wire. The mechanical hydraulically boosted flight control system utilizes mechanical linkages to control a hydraulic servo actuator instead of direct connection to the control surfaces. For the fly-by-wire systems either an analog circuit or a digital computer is used to sense pilot control inceptor inputs and apply a command to a servo actuator to drive the control surfaces. The flyby-wire and a mechanical hydraulically boosted control system are examples of irreversible flight control systems. For both systems the aerodynamic forces cannot be felt by the pilot because of the absence of the direct linkage to the control surfaces. For these systems, an artificial feel system is often implemented to simulate any force feedback from flight control characteristics (Takats, 2011). An example of a fly-by-wire flight control system is shown in Figure $1 \mathrm{~b}$ ).

In Figure $1 \mathrm{~b}$ ), the deflection of the control inceptor (1) from the operator is analyzed by an analog or digital computer. The computer then sends the proper command to a hydraulic valve actuator (6) which provides the hinge moment to deflect the control surface (5). An autopilot system (2) can bypass the control by the operator by directly controlling the command to the hydraulic valve actuator (6).

\section{B. Flight Simulator Control Dynamics}

Control loader systems are utilized in simulators to simulate the flight control system characteristic dynamics. These dynamics include the force gradient, damping, natural frequency, friction, detents, breakout force, and any other nonlinear dynamics. Early control loader systems used a position feedback control loop. An example of a position feedback control loader system in shown in Figure 2 a). As the control inceptor is moved, both the force and position on the control inceptor is sensed. The force is fed through the aft mass and forward mass simulations which creates the appropriate dynamics. The combination of the aft and forward mass simulation results in a commanded position. The servo drive command is produced when the position of the control inceptor is subtracted from the commanded position. The position servo control loader limited the achievable bandwidth. Therefore, a force loop was implemented to obtain a higher bandwidth and because human subjects predominantly use force to control a vehicle (Takats, 2011).

The force feedback control loader (Figure $2 \mathrm{~b}$ )) uses a force command to drive the servo. As the control inceptor is deflected, both the position and force are sensed. The position of the control inceptor is implemented through the forward mass, aft mass, and cable simulations to produce a force command to the servo valve. The servo drive command is produced when the force of the control inceptor is subtracted from the calculated commanded position. A control loader system commands the control inceptor to the proper position based on the force applied by the operator. 


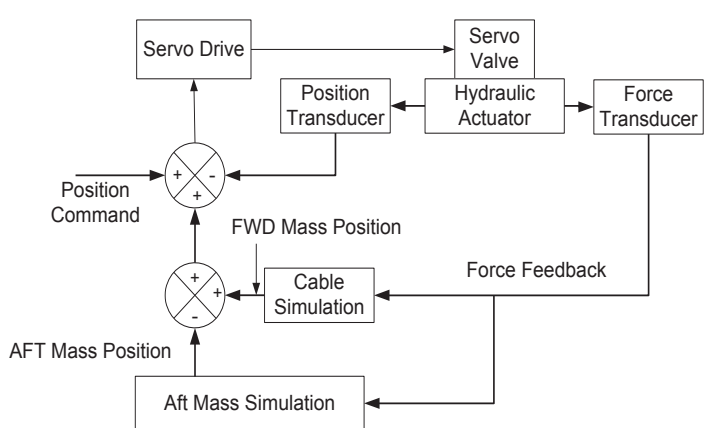

Figure 2 a) Position Servo Control Loader Model (Takats, 2011)

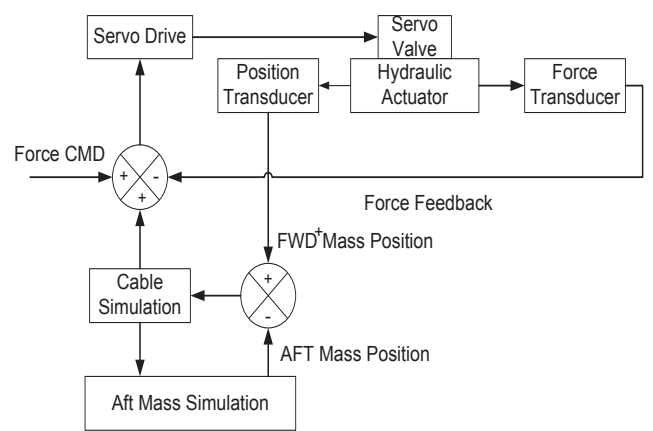

b) Force Servo Control Loader Model (Takats, 2011)

\section{The Neuromuscular System}

The neuromuscular system is an important component in the control of a vehicle. The visual, vestibular, and somatosensory system give feedback to the operator relaying important information regarding vehicle state. The sensory information is processed by the central nervous system and a command is applied to the neuromuscular system. The output of the neuromuscular system is a force. The neuromuscular system has its own internal feedback loop. The golgi tendons and muscle spindle relay information on the muscle extension and force applied by the muscles to the limbs. This allows for course as well as fine motor control of a task. This allows the pilot to be adaptable and able to control many varying dynamic tasks. One question brought up by the study of the neuromuscular system is the limitation of the neuromuscular system and the situations that cause a task to be uncontrollable.

Don R. Gum (1973) proposed a lateral head motion model (Figure 3). This model includes the muscle dynamics, head dynamics, and muscle spindle feedback. The transfer function for the muscle is defined as a first order system. The muscle spindle is represented by a lead/lag and the head dynamics is represented by a typical spring mass damper second order system.

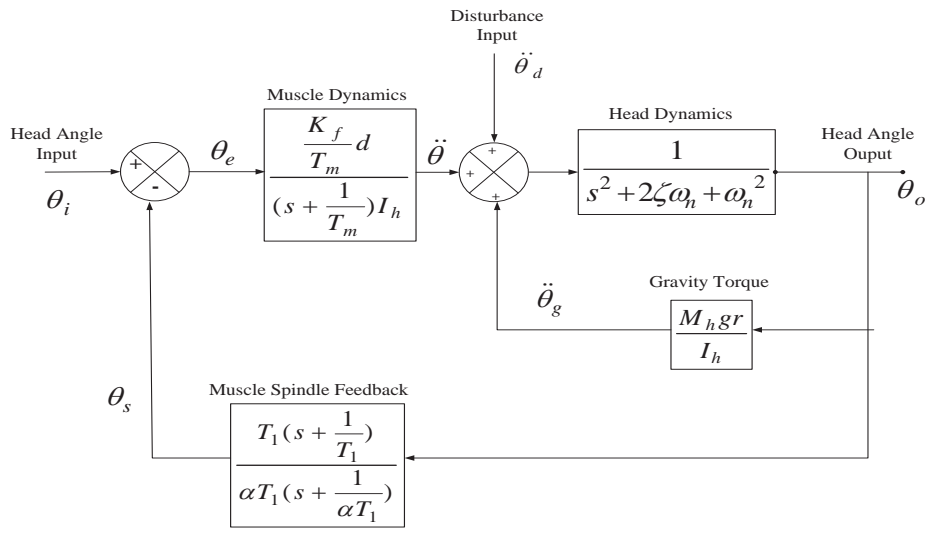

Figure 3 Lateral Head Motion Block Diagram (Gum, 1973)

In the pursuit of developing pilot models, R.E. Magdaleno and D.T. McRuer (1971) investigated many aspects of the neuromuscular system and proposed similar neuromuscular models. One such model is the neuromuscular subsystems model which was developed using subject describing functions based on an input/output relationship (Figure 4). To obtain a specific model for the muscle and muscle spindle feedback, electromyographic (EMG) signals were recorded from each subject. This allowed for the measurement of the effective muscle activation signal and average tension in the muscle. 


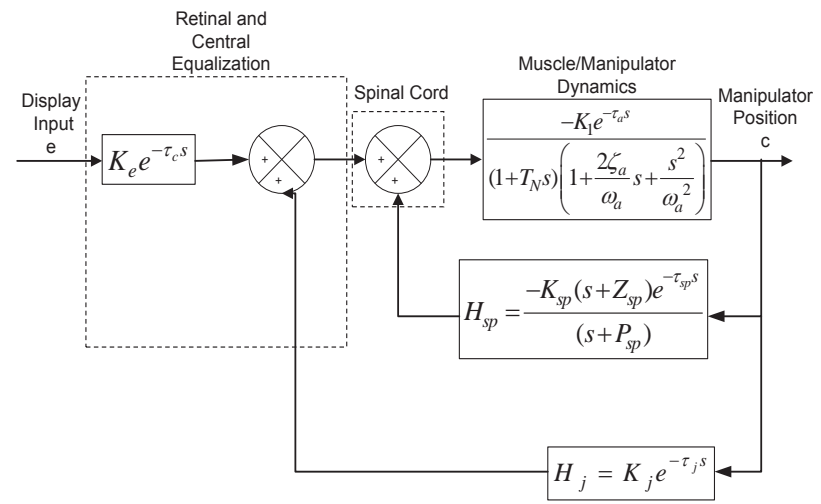

Figure 4 Neuromuscular Subsystems Model (Magdaleno \& McRuer, 1971)

In developing a structural model of a pilot (Figure 5), Ronald Hess (1985) employed unknown parameters of the neuromuscular system which were based on the crossover model matched to pilot input/output relationships for various tasks. He uses the crossover model to equalize the structural model and chose parameters to have K/s dynamics around $2 \mathrm{rad} / \mathrm{s}$.

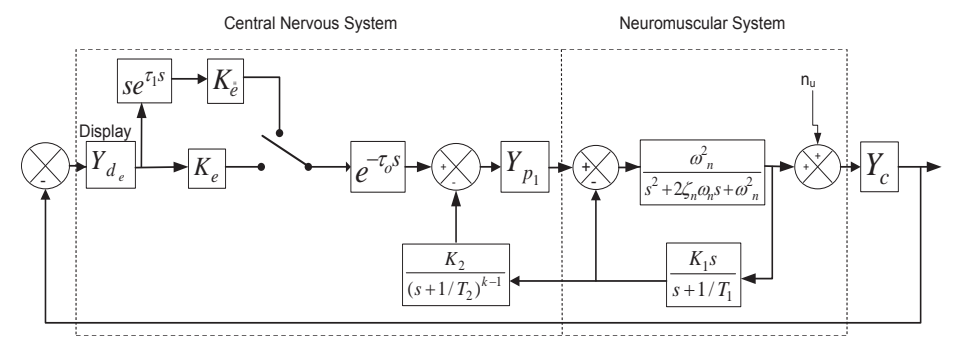

Figure 5 The Structural Model of the Human operator for Compensatory Tracking (Hess, 1985)

Figure 6 illustrates a neuromuscular model that has recently been proposed by Ruud Hosman and David Abbink (Hosman, 2010). This model also takes a structural approach by using subject input/output relationships to define the parameters.

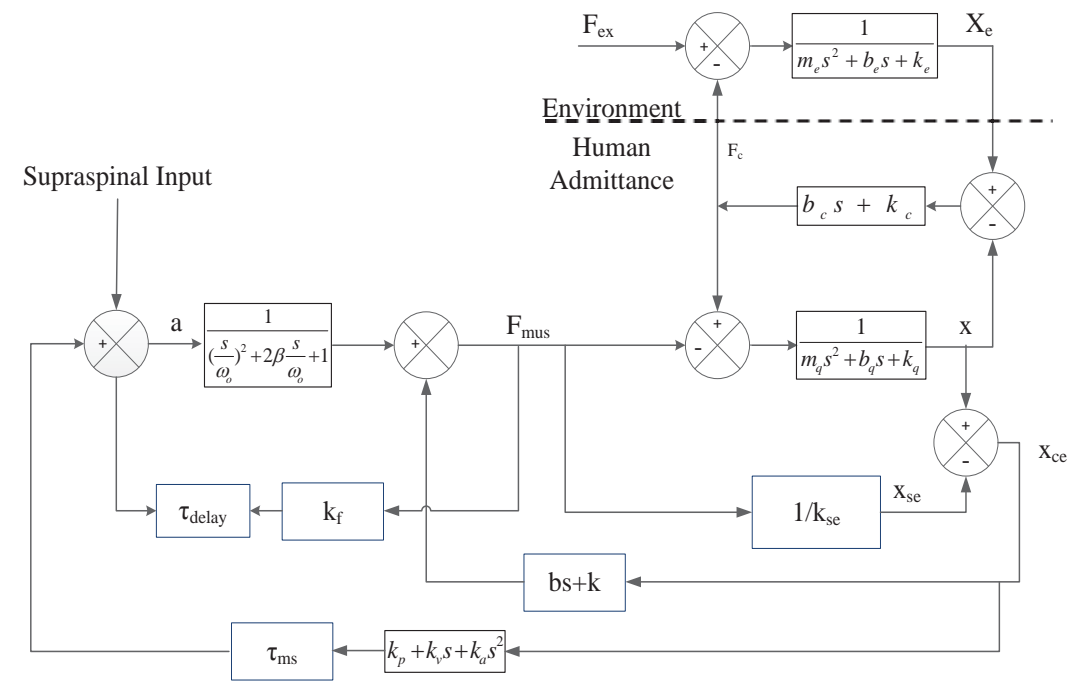

Figure 6 Neuromusculoskeletal Model (Hosman, 2010) 


\section{Transfer of Training}

Transfer of training is a task wherein certain skills and performance attributes are learned by subjects in a training phase and then subjects will apply the learned skills to a new situation. In a transfer of training experiment a subject is trained in a simulator and then transferred to the real world vehicle. By comparing how well the subjects perform in both the simulator and the real world vehicle, the training effectiveness can be measured. A minimum of two different subject groups are used to test training advantage. One group is trained on a high fidelity simulation and another group is trained on a simulation with certain variables changed. The two groups will then transfer to the real world vehicle.

A variation of a transfer of training study is a quasi-transfer of training study. Since using a real world vehicle is cost ineffective and time consuming, a quasi-transfer of training study can be conducted. A quasi-transfer of training paradigm differs in that a simulator is used to test both training and transfer tasks. One group will train on a high fidelity simulator and another group will train on a simulator with certain variables altered. The two groups will then transfer to the high fidelity simulator which represents the real world vehicle. A quasi-transfer of training study is easily replicable and can be performed for a wide variety of tasks.

An example of a quasi-transfer of training study is an experiment conducted by William H. Levison (1979). Five groups of subjects trained on a roll-axis tracking tasks. One group trained with only visual cues. Another group trained with combined synchronous visual and motion cues. The other groups trained with motion cues delayed in relation to visual cues by 80, 200, and 300 msec. The motion cues were provided by a Roll Axis Tracking Simulator (RATS) at the Aerospace Medical Research Laboratory. One group trained with no motion and then transferred to the RATS with synchronized motion and visual cues turned on. Another group trained with the synchronous motion and visual turned on in the RATS and then transferred to the synchronous motion and visual simulation in the RATS. The groups that trained on the delayed motion cues, trained in the RATS with motion delayed 80, 200 and 300 msec and then transferred to the synchronous motion and visual simulation in the RATS. The subjects all trained to an asymptotic mean square or root mean square tracking error before transferring to the synchronous motion and visual simulation.

\section{A Quasi Transfer of Training Study}

A quasi transfer of training study, in which high fidelity and low fidelity force feedback dynamics are interchanged, is conducted in order to develop an understanding of the prominence of force feedback cues to the operator. The training effectiveness of high fidelity versus low fidelity control loading system has not been hitherto studied. The study presented in this paper attempts to illuminate these effects. A study was designed employing a disturbance compensatory roll tracking task with $1 / \mathrm{s}$ and $1 / \mathrm{s}^{2}$ plant dynamics. The quasi transfer of training study consists of training subjects to null a roll disturbance using a high and low fidelity control loader simulation. The roll disturbance compensation task used in the experiment is described by the block diagram of Figure 7. A disturbance consisting of a sum of 9 sinusoids (Table 1) deflects a bank angle line viewed on the display.

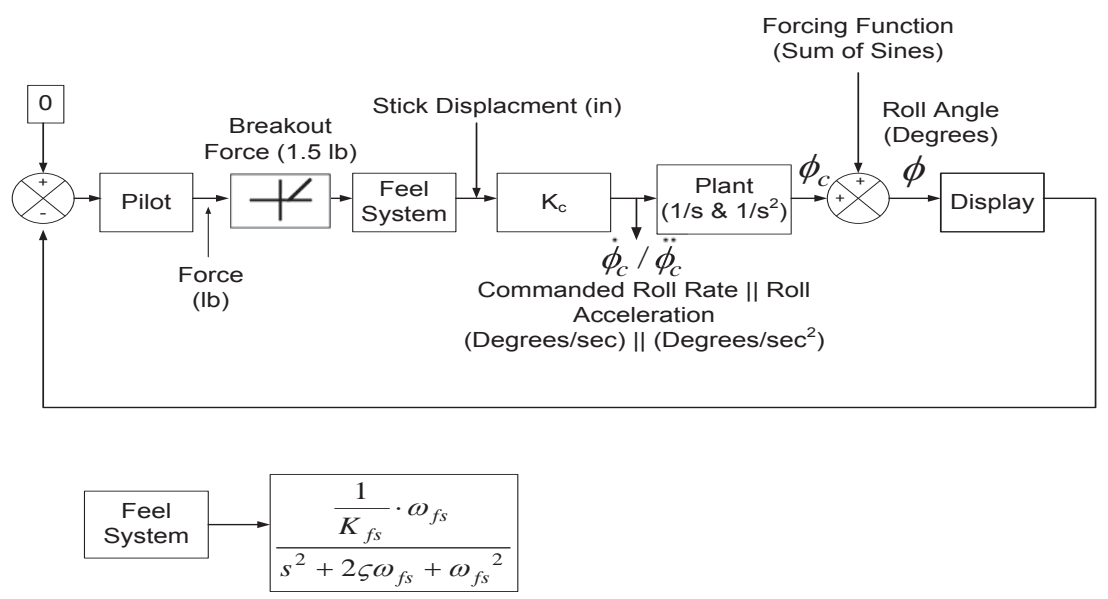

Figure 7 Developed Roll Disturbance Compensatory Tracking Task 


\begin{tabular}{|c|c|c|c|c|c|c|c|c|c|c|}
\hline Plant Dynamics & Sine Wave & 1 & 2 & 3 & 4 & 5 & 6 & 7 & 8 & 9 \\
\hline $1 / \mathrm{s} \& 1 / \mathrm{s}^{2}$ & Frequency $\left(\omega_{\mathrm{i}} \mathrm{rad} / \mathrm{s}\right)$ & .467 & .701 & 1.17 & 1.87 & 3.51 & 7.01 & $11 / 2$ & 14 & 18.7 \\
\hline & Amplitude $\left(\mathrm{A}_{\mathrm{i}} \mathrm{deg}\right)$ & 15.2 & 15.2 & 15.2 & 7.6 & 3.04 & .76 & .38 & .228 & .152 \\
\hline
\end{tabular}

Table 1 Sum of Sines Disturbance Input (Adapted from Johnston, 1988)

The commanded plant input is equal to the subject control inceptor deflection multiplied by a roll rate or roll acceleration per inceptor deflection command gain $\left(K_{c}\right)$ for $1 / s$ and $1 / s^{2}$ plant dynamics respectively. The commanded plant input is then passed through plant dynamics to produce a roll angle. The roll angle of the vehicle is displayed as an angle displaced from an artificial horizon line representing level flight. The visual feedback and proprioceptive feedback are the main cues to the subject, in order to null disturbance and maintain an angle of zero degrees from the horizon line.

The displayed roll angle causes the person performing the compensatory task to output a force from muscle activation on the control inceptor. The deflection of the control inceptor in inches is multiplied by a roll command gain $\left(\mathrm{K}_{\mathrm{c}}\right)$. The roll command gain converts the deflection of the control inceptor to a roll rate command $\left(\dot{\phi}_{c}\right)$ and roll acceleration command ( $\ddot{\phi}_{c}$ ) for $1 / \mathrm{s}$ and $1 / \mathrm{s}^{2}$ plant dynamics respectively. The roll rate command ( $\dot{\phi}_{c}$ ) and roll acceleration command $\left(\ddot{\phi}_{c}\right)$ are displayed as a roll angle $(\phi)$ after being passed through $1 / \mathrm{s}$ and $1 / \mathrm{s}^{2}$ dynamics respectively.

The roll command gain $K_{c}$ is set to a value of $40 \mathrm{deg} / \mathrm{s} \bullet$ in and $40 \mathrm{deg} / \mathrm{in} \bullet \mathrm{s}^{2}$ for plant dynamics of $1 / \mathrm{s}$ and $1 / \mathrm{s}^{2}$ respectively. When the deflection output of the control inceptor in inches is multiplied by the roll command gain it creates a roll rate given in $\mathrm{deg} / \mathrm{s}$ and roll acceleration in $\mathrm{deg} / \mathrm{s}^{2}$ for $1 / \mathrm{s}$ and $1 / \mathrm{s}^{2}$ dynamics respectively.

\section{Determination of Force Feel Parameters}

The control loader system used in the experiments is a force feedback control loader system developed by McFadden Simulator Systems. The block diagram of its internal control structure is located in Figure 8. When the subject applies a force, the position of the control inceptor is fed back through the implemented dynamics. The parameters that create the force feel dynamics consist of the forward damping, the force gradient, the breakout force, and forward stop force. The position is fed through the force gradient to produce a force command which could be non-linear. The velocity of the control inceptor is fed through the damping coefficient to also produce a force due to the damping. The breakout force applies a high force gradient and a certain force level around the neutral position of the control inceptor. The control inceptor will not move until the breakout force level is reached. The forward stop position sets the max deflection of the control inceptor in either direction.

The force that is applied to the control inceptor by the operator is first summed with the force command developed from the characteristic forward mass dynamics to produce an applied force. The applied force is then subtracted from the measured force on the control inceptor, which produces a force error command that drives the servo to the correct position. A force can be developed by a computer or analog circuitry and then injected in the control loader system at the same summing junction where operator force input is usually added. This allows for frequency sweep and step response data to be applied to the control loader system.

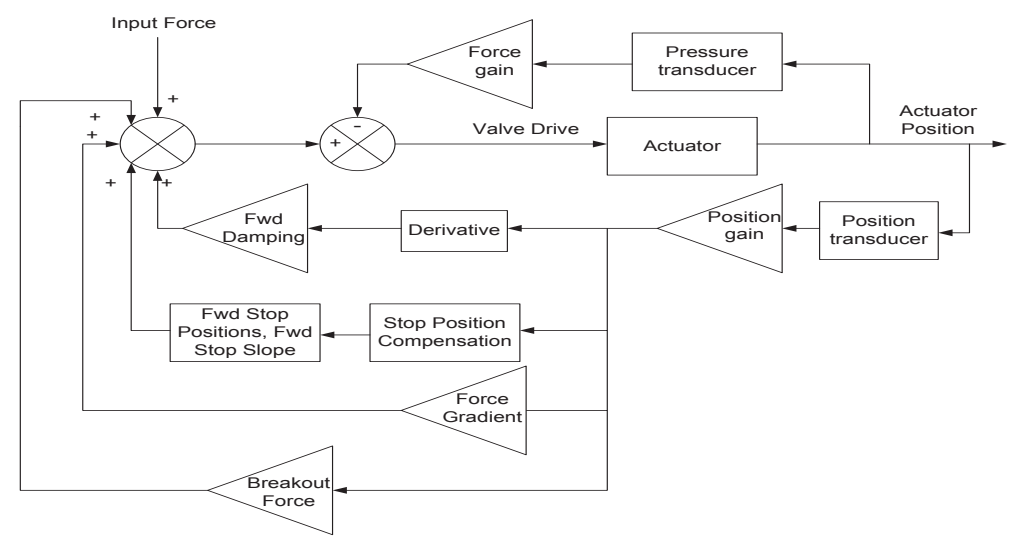

Figure 8 McFadden Control Loader Model 
The high fidelity control loader parameters were adopted from the literature. These parameters were determined to produce a good task performance from test subjects within the many experiments conducted in the literature. Two of the characteristics that determined good flying performance are subject tracking error and tracking bandwidth. Throughout the literature, many experiments were conducted in which different control inceptor characteristics were tested. The parameters were chosen that produced the smallest tracking error, and the highest tracking bandwidth (Bailey 1990; Johnston, 1988; Mitchell, 1992). The parameters that defined a high fidelity control inceptor are a force gradient of $4 \mathrm{lb} / \mathrm{in}$, a damping ratio of 0.7 , a natural frequency of between 14 and 26 $\mathrm{rad} / \mathrm{s}$, and a breakout force of $1.5 \mathrm{lb}$. The transfer function used to simulate the high fidelity control inceptor is shown in Equation 1. The term (X) and (F) represents the lateral control inceptor deflection and input force respectively. A step response of the high control inceptor with a natural frequency set to $26 \mathrm{rad} / \mathrm{s}$ is shown below in Figure 9. The simulated frequency response of the high fidelity control loader is shown below in Figure 10.

$$
\frac{X}{F}=\frac{(26)^{2}}{s^{2}+2(.7)(26)+(26)^{2}}
$$

\section{Equation 1}

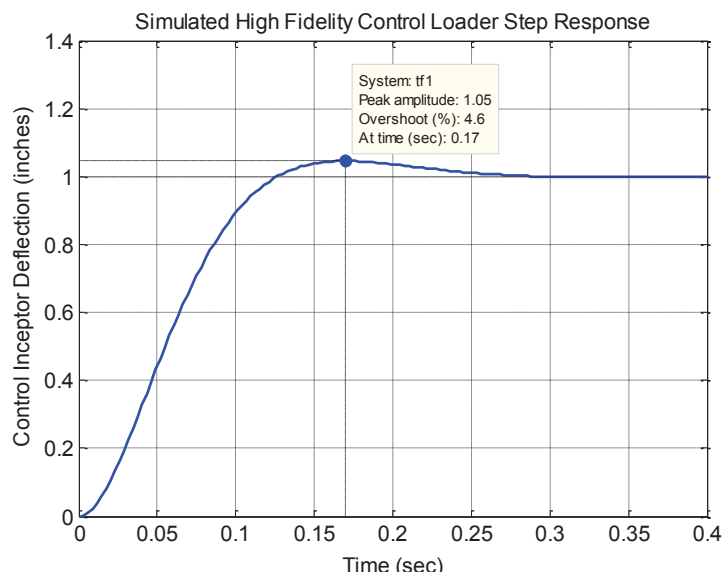

Figure 9 Simulated High Fidelity Control Loader Step Response
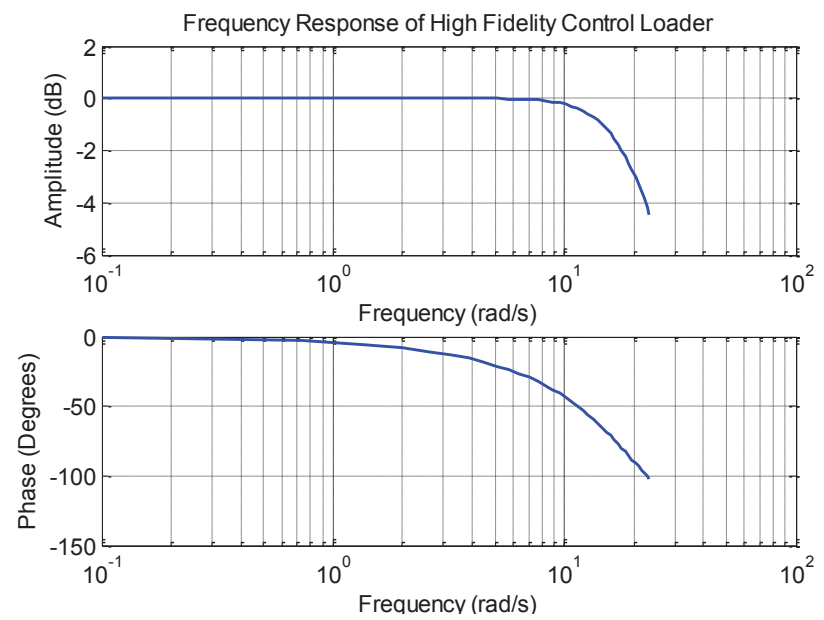

Figure 10 Simulated High Fidelity Control Loader Frequency Response 
The low fidelity parameters were determined from a Logitech Force 3D Pro which is used for most flight simulator games. The parameters measured about the roll-axis of the controller are a natural frequency of $34.5 \mathrm{rad} / \mathrm{s}$, a damping ratio equal to 0.135 , and a force gradient of $0.7 \mathrm{lb} / \mathrm{in}$. The damping ratio and corresponding natural frequency were found using the log decrement. To simulate the step response and frequency response for the low fidelity control loader the transfer function in Equation 2 is utilized. The natural frequency of $8.366 \mathrm{rad} / \mathrm{s}$ in Equation 2 was obtained using a force gradient of $0.7 \mathrm{lb} / \mathrm{in}$ and the McFadden control inceptor mass of $0.01 \mathrm{lb}^{\circ} \mathrm{s}^{2} / \mathrm{in}$. The natural frequency used in the simulated transfer function represents the natural frequency expected from the McFadden control loader. Since the mass of the McFadden control loader is greater than that of the Logitech Force 3D Pro computer joystick, the expected natural frequency is smaller. The simulated step response (Figure 11) and frequency response (Figure 12 ) for the low fidelity control represents the characteristic response estimated when only the force gradient and damping ratio found from the Logitech Force 3D Pro computer joystick is implemented. Table 2 summarizes the input parameters for the McFadden control loader.

$$
\frac{X}{F}=\frac{(8.366)^{2}}{s^{2}+2(.135)(8.366)+(8.366)^{2}}
$$

\section{Equation 2}

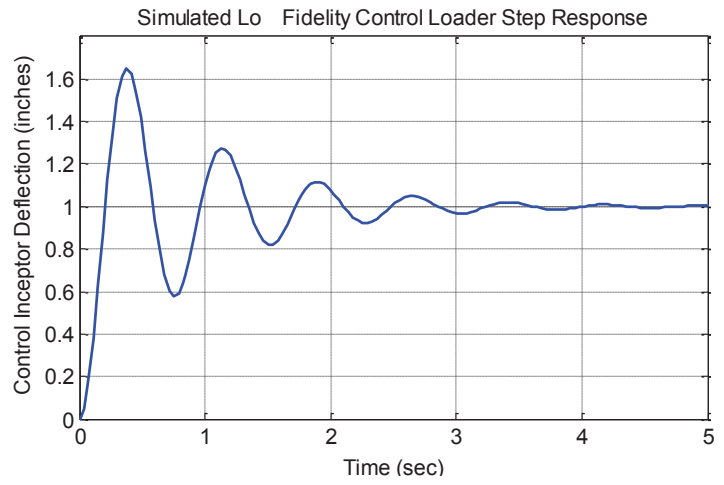

Figure 11 Simulated Low Fidelity Control Loader Step Response
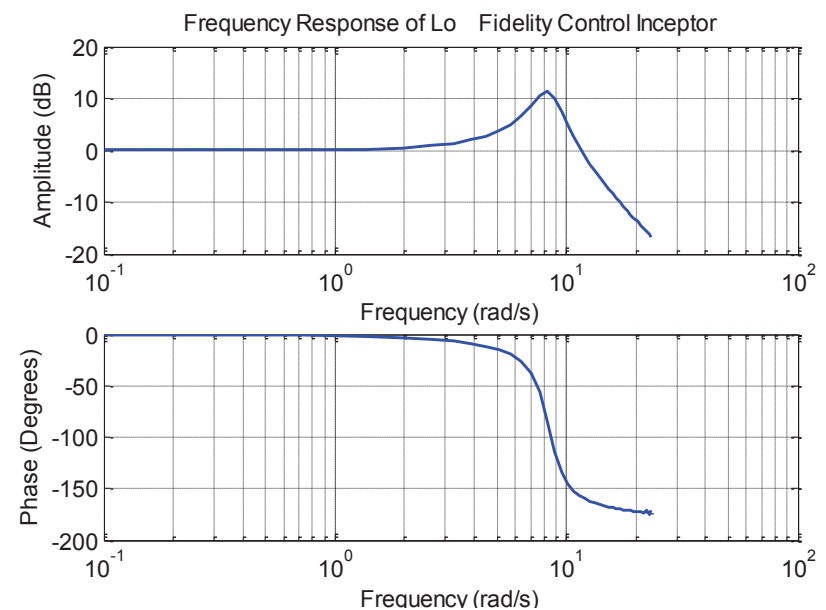

Figure 12 Simulated Low Fidelity Control Inceptor Frequency Response

\begin{tabular}{|c|c|c|c|}
\hline Control Inceptor Fidelity: & Force Gradient: & Damping Ratio $\zeta$ & Breakout Force: \\
\hline High Fidelity & $4 \mathrm{lb} / \mathrm{in}$ & .7 & $1.5 \mathrm{lb}$ \\
\hline Low Fidelity & $.7 \mathrm{lb} / \mathrm{in}$ & .135 & $0 \mathrm{lb}$ \\
\hline
\end{tabular}

Table 2 Control Inceptor Characteristics 


\section{Analysis of Force Feel Parameters}

To verify that the control loader parameters are implemented correctly, a force gradient, frequency response and free release response of the control loader dynamics for both the high fidelity and low fidelity system were measured. The first measured characteristic is the force gradient obtained by plotting the force applied versus the displacement of the control inceptor (Figure 13).

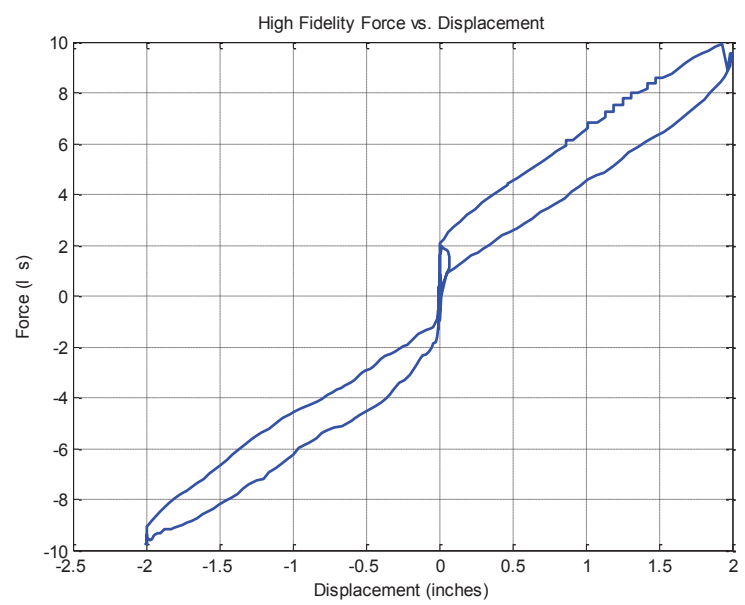

Figure 13 High Fidelity Control Inceptor Force vs. Displacement

As shown in Figure 13, the control inceptor contains hysteresis. The control inceptor follows a different force vs. displacement path when being pulled as opposed to being pushed. The force gradient obtained from the slope in Figure 13 is equal to $4.09 \mathrm{lb} / \mathrm{in}$, thereby verifying the implementation. At zero inches of deflection, there exists a non-linearity, which is the breakout force. As shown, the breakout force is approximately $1.5 \mathrm{lb}$.

The free release response was conducted for the high fidelity control inceptor with an implemented damping coefficient of $0.0243 \mathrm{lb} \bullet /$ in which corresponds to the specified 0.7 damping ratio. A free release test is conducted by deflecting the control inceptor to its maximum deflection in one direction and then releasing. The free release response is shown below in Figure 14. As shown, the response contains a small amplitude peak and settles fairly quickly. The overshoot for this system is equal to $4.022 \%$ which corresponds to a damping ratio of 0.715 . Because of friction, the measured damping ratio is slightly larger than specified.

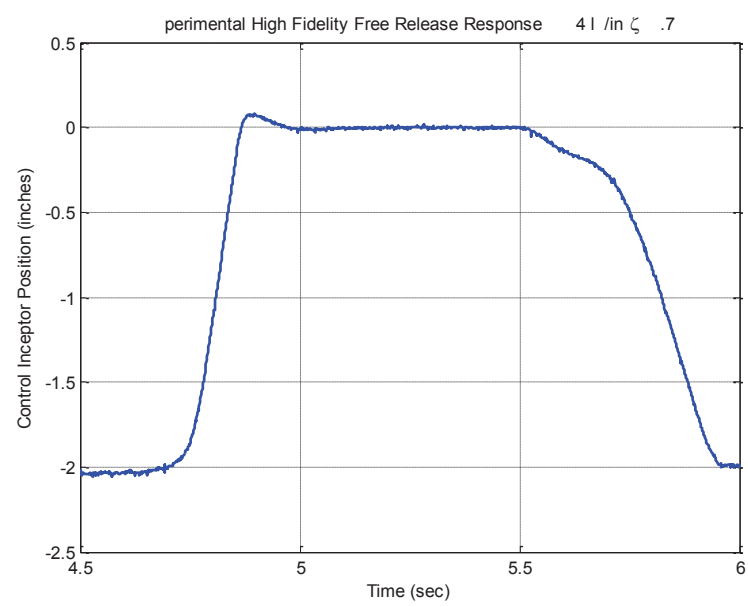

Figure 14 Experimental High Fidelity Free Release Response 
For the frequency response test a frequency sweep was provided as the input representing a force command to the force servo of the control loader. The output is the measured force from the force transducer of the McFadden control loader.

The damping coefficient was lowered to $0.01 \mathrm{lb} \bullet \mathrm{s} / \mathrm{in}$ to obtain a free release response with multiple oscillations. This was used to calculate an effective mass. A damping ratio 0.118 was calculated from the free release response with the damping coefficient set to $.01 \mathrm{lb} \bullet \mathrm{s} / \mathrm{in}$. The damping coefficient was kept at $.01 \mathrm{lb} \bullet \mathrm{s} / \mathrm{in}$ when simulating the frequency response of the high fidelity control loader. Using the obtained force gradient of 4.09 $\mathrm{lb} / \mathrm{in}$ and an effective mass of $0.0119 \mathrm{lb} \cdot \mathrm{s}^{2} / \mathrm{in}$, the calculated natural frequency is equal to $18.54 \mathrm{rad} / \mathrm{s}$. A damping ratio of 0.118 along with a natural frequency of $18.54 \mathrm{rad} / \mathrm{s}$ is used when simulating the frequency response. Using the signal processing technique the following high fidelity control loader frequency response is shown in Figure 15.
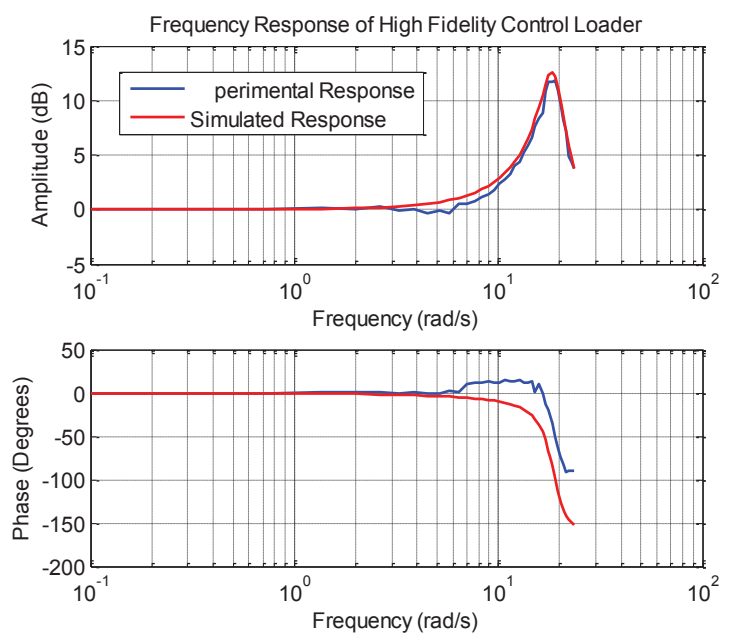

Figure 15 Experimental High Fidelity Frequency Response with Low Damping Ratio

The same tests performed above for the high fidelity control inceptor were performed for the low fidelity control loader. The force vs. displacement test is shown in Figure 16.

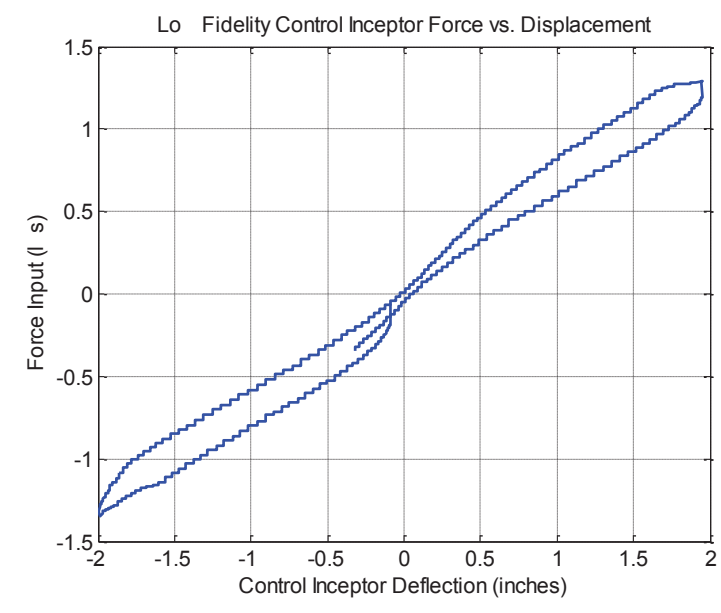

Figure 16 Experimental Low Fidelity Force vs. Displacement

The mean slope of the low fidelity control loader force vs. displacement curve was found to be .66 lb/in which is $0.04 \mathrm{lb} /$ in different than the defined value of $0.7 \mathrm{lb} / \mathrm{in}$. The non-linearity at 0 inches of deflection that is present for the high fidelity control inceptor is not present for the low fidelity control inceptor, since the breakout force is set to $0 \mathrm{lb}$. There still exists hysteresis for the low fidelity control loader. 
The free release response was also conducted for the low fidelity control loader and is shown in Figure 17. As shown, the free release response represents a very lightly damped system. The overshoot of $48.2 \%$ corresponds to a damping ratio of 0.226 . Using the log decrement on the first two successive peaks we obtain a damping ratio of 0.233 . The defined damping ratio is equal to 0.135 but because of added friction to the system, the damping ratio is larger than what was defined. The natural frequency obtained from the free release response is equal to $5.26 \mathrm{rad} / \mathrm{s}$.

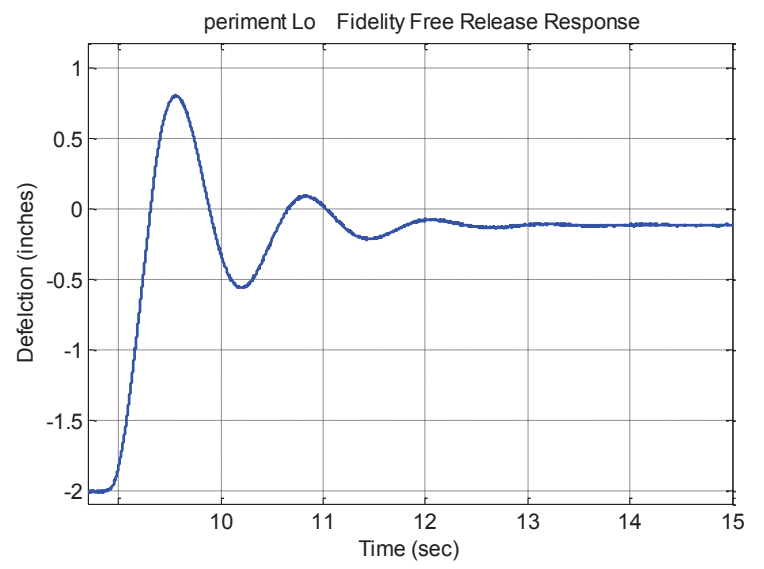

Figure 17 Experimental Low Fidelity Free Release Response

Both the experimentally obtained frequency response and a simulated response are shown in Figure 18. A damping ratio of 0.226 and a natural frequency of $5.26 \mathrm{rad} / \mathrm{s}$ that are both obtained from the free release response (Figure 17) are used to simulate the frequency response. The peak magnitude of the simulated and experimental frequency response is equal to 7.125 and $7.4 \mathrm{~dB}$ respectively. The peak magnitude corresponds to the damping ratio of the system. The simulated peak magnitude corresponds to a damping ratio of 0.226 . A peak magnitude of $7.4 \mathrm{~dB}$ corresponds to a damping ratio of 0.219 . Therefore the difference is equal to $3 \%$.
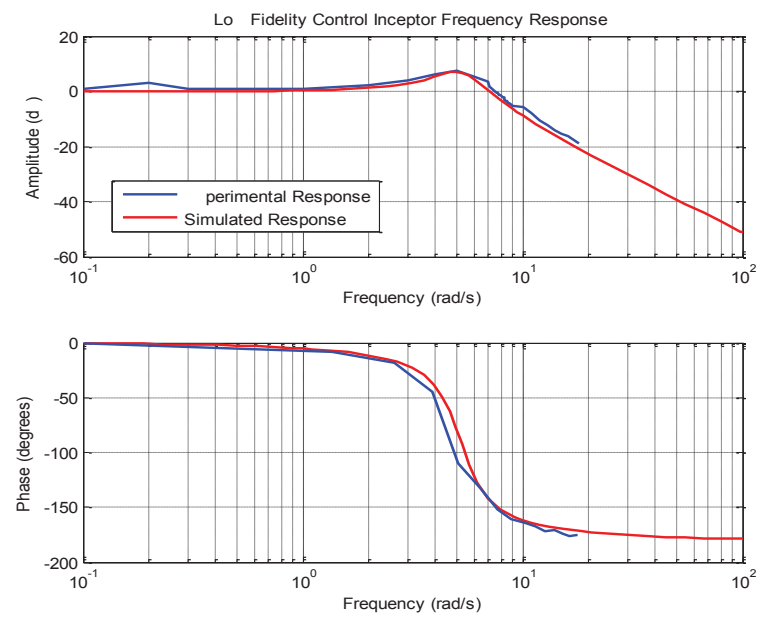

Figure 18 Experimental Low Fidelity Frequency Response

The peak magnitude for the simulated and experimental response occurs at $5 \mathrm{rad} / \mathrm{s}$ and $4.965 \mathrm{rad} / \mathrm{s}$ respectively. The natural frequencies that correspond to the peak natural frequencies for the simulated and experimental responses are equal to $5.26 \mathrm{rad} / \mathrm{s}$ and $5.24 \mathrm{rad} / \mathrm{s}$ respectively. The difference between these two natural frequencies is equal to $.4 \%$.

The expected natural frequency for this system using the effective mass of the control loader of .0119 $\mathrm{lb} \bullet \mathrm{s}^{2} /$ in and a force gradient of .7 lb/in is equal to $7.67 \mathrm{rad} / \mathrm{s}$. The control loader contains friction which effectively damps the system which acts as an added mass. The calculated effective mass for the low fidelity control loader is equal to $.025 \mathrm{lb} \cdot{ }^{2} /$ in. The effective mass for the low fidelity control inceptor is $.0131 \mathrm{lb} \cdot \mathrm{s}^{2} /$ in greater than the high fidelity case. 


\section{Experimental Setup}

The experiments of this study were conducted in the Generic Flight Deck (GFD) simulator located in the Cockpit Motion Facility (CMF) at NASA Langley Research Center. The motion platform has six degrees of freedom which includes six-76 inch hydraulic actuators. The facility allows the use of each one of the three flight cockpits with motion or as a fixed base simulation. The flight cockpits used in a fixed based simulation are located on either side of the Cockpit Motion Facility. The three flight cockpits include the Research Flight Deck Simulator (RFD), the Integration Flight Deck Simulator (IFD), and the Generic Flight Deck Simulator (GFD). The GFD cockpit was used as a fixed based simulation for the experiments performed within this paper.

To conduct the transfer of training task, two separate groups each consisting of 10 test subjects were used. The two groups represent the high fidelity control inceptor transfer group and the low fidelity control loader transfer group. The high fidelity control loader transfer group trained on the high fidelity control loader simulation. The low fidelity control inceptor transfer group trained on the low fidelity control inceptor simulation. The test subjects each conduct a 30 second run. The RMS tracking error and RMS control inceptor deflection were recorded for each run. The RMS cumulative running average was computed for both the tracking error and control inceptor deflection.

When each test subject reaches an asymptotic running cumulative average RMS tracking error, the training task will be completed for that subject. To assist in determining if the subject has reached asymptote is the slope of the running cumulative average RMS tracking error between every two points. When the slope reaches a consistent value for three consecutive runs of 0.1 or less, asymptote has been reached. After each subject trains, they will then be transferred to the high fidelity control loader simulation. The test subjects will again complete 30 second runs until they reach an asymptotic running cumulative average (RMS) tracking error.

The subjects performed four phases of the experiment that include the familiarization phase, evaluation phase, training phase, and transfer phase. The subject will then transfer to the high fidelity control loader simulation. After they have completed the task for $1 / \mathrm{s}$ plant dynamics, they returned to repeat the task for $1 / \mathrm{s}^{2}$ plant dynamics.

For this experiment, a variety of parameters were recorded. Some of the parameters to be measured include pilot horizontal control inceptor deflection, horizontal control inceptor force, and tracking error. Some of the calculated parameters include the running cumulative average RMS tracking error, the slope of the average RMS tracking error, RMS control inceptor deflection, and PSD of the control inceptor deflection. One of the most important parameters is the RMS tracking error, which is used to determine if the subject has reached a performance asymptote. To calculate the RMS tracking error, the bank angle is recorded. Each data point is squared and summed together then divided by the total number of data points. This is represented in Equation 3.

$$
x_{r m s}=\sqrt{\frac{x_{1}^{2}+x_{2}{ }^{2}+x_{3}{ }^{2}+\cdots+x_{n}{ }^{2}}{n}}
$$

Equation 3

The term ( $\mathrm{x}_{\mathrm{rms}}$ ) represents the RMS of any data that are recorded. The symbol "n" represents the total number of data points. The term $\left(\mathrm{x}_{\mathrm{n}}\right)$ represents each data point recorded at a discrete time interval. To calculate the running cumulative average of the RMS tracking error, Equation 4 can be used.

$$
\bar{x}_{r m s}(j)=\frac{(j-1) \cdot \bar{x}_{r m s}(j-1)+x_{r m s}(j)}{j}
$$

Equation 4

The term $\mathrm{j}$ represents the run number. The term $\bar{X}_{r m s}(j)$ and $\bar{X}_{r m s}(j-1)$ represents the current RMS running cumulative average and the previous RMS running cumulative average respectively. The term $x_{r m s}(j)$ represents the current run RMS tracking error. The RMS running cumulative average has to be initialized to the RMS of the first run. The averaging technique in Equation 4 is applied on the second run. The running cumulative average of the RMS tracking error for the first run is just the RMS tracking error of the first run.

To determine if the RMS running cumulative average has reached an asymptote, the slope between every two runs will be calculated. As the slope between two runs reaches 0.1 for 3 consecutive runs, asymptote has been reached. The formula for the slope between two runs is listed in Equation 5.

$$
\Delta \bar{x}_{r m s}(j)=\bar{x}_{r m s}(j)-\bar{x}_{r m s}(j-1)
$$

Equation 5

13

American Institute of Aeronautics and Astronautics 
The term $\Delta \bar{X}_{r m s}(j)$ represents the slope of the running cumulative average RMS between two runs. The terms $\bar{x}_{r m s}(j)$ and $\bar{x}_{r m s}(j-1)$ represent the current and previous run RMS tracking error respectively.

The display consists of a horizon line and a bank angle reference line that will displace at an angle from the horizon reference line (Figure 19). The image will be displayed on an out the window display. The background is black and the horizon reference line is white. The roll reference line is green in color to distinguish it from the horizon reference line. The geometry of the displayed scene was calculated from Equation 6 and Equation 7. The term HFOV represents the horizontal field of view angle, and $\mathrm{D}_{\mathrm{EP}}$ is the nominal distance from the display screen that the eye of the subject is located.

$$
\begin{aligned}
& \mathrm{Hv}=2 \cdot \tan (\mathrm{HFOV} / 2) \cdot \mathrm{D}_{\mathrm{EP}} \\
& \mathrm{Vv}=2 \cdot \tan (\mathrm{VFOV} / 2) \cdot \mathrm{D}_{\mathrm{EP}}
\end{aligned}
$$

Equation 6

Equation 7

The term VFOV represents the vertical field of view angle. The length of the horizon reference line should be $100 \%$ of the horizontal viewing dimension. The length of the bank angle reference line should be approximately $80 \%$ of the vertical viewing dimension. The dimensions of the target on the display are shown in Figure 19. These dimensions are calculated using a vertical FOV of $27^{\circ}$, a horizontal FOV of $44^{\circ}$, and an eye point distance from the collimator of 50 inches.

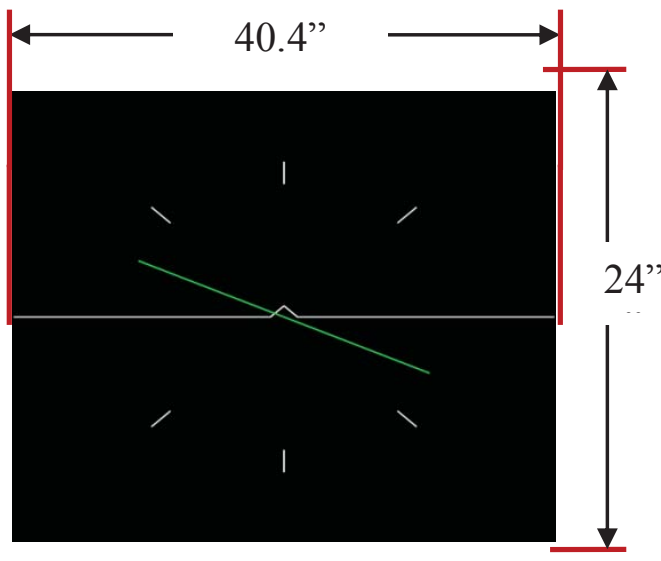

Figure 19 Out the Window Display

\section{Discussion of Results}

Shown below in Figure 20 are the aggregate results for the high fidelity training group with $1 /$ s plant dynamics. This figure is divided into the training phase represented by the blue curves and the transfer phase represented by the red curves. The solid line represents the average tracking error across the subjects within the high fidelity group. The dotted line represents one standard deviation $(\sigma)$ in either direction. As shown, the tracking error reaches an asymptote both in the training and transfer phases. During the training phase the most improvement is shown with the average tracking error for the high fidelity training group improving 4.13 degrees over 25 runs. The asymptotic tracking error for the training phase is equal to 11.94 degrees. When the subjects transferred, the tracking error began at 12.49 degrees and then settled to 11.58 degrees. This is approximately a $3 \%$ difference from the asymptotic tracking error in the training phase. Subjects were able to train to a certain performance level and then maintain this in the transfer phase. Also shown in Figure 20, the standard deviation of the average tracking error among subjects in the training phase was larger than for the transfer phase.

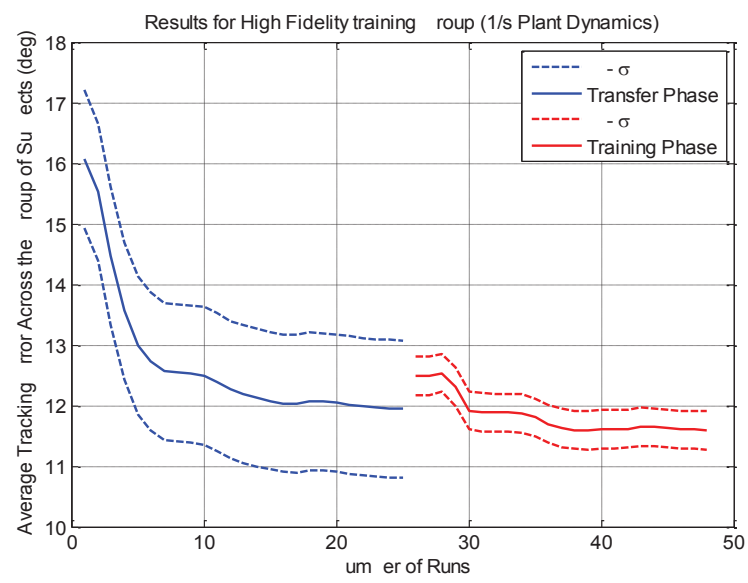

Figure 20 Results for High Fidelity Training Group (1/s) 
Shown in Figure 21 are the corresponding results for the low fidelity training group for 1/s plant dynamics. The low fidelity training group had an initial tracking error that was higher than for the high fidelity training group. The initial average tracking error across subjects is equal to 21.06 degrees, which is $31 \%$ higher than the corresponding high fidelity training group value. As shown, the asymptotic tracking error for the training phase is equal to 15.79 degrees. When the low fidelity training group transfers to the high fidelity control loader simulation, the initial tracking error increases from the asymptotic value in the training phase. This is expected, since the dynamics of the high fidelity control loader are unknown to the subjects. The subjects quickly adapt and they are able to reach an asymptotic tracking error approximately $13 \%$ lower than the training asymptotic tracking error. The final average tracking error between subjects reaches a value of 13.7 degrees, which is $16 \%$ greater than the high fidelity training group. The standard deviation increases as the low fidelity training subjects transfer to the high fidelity control loader simulation.

Figure 22 illustrates the subject results for the high fidelity group with $1 / \mathrm{s}^{2}$ plant dynamics. The average tracking error across the group of subjects is higher for the task with $1 / \mathrm{s}^{2}$ plant dynamics. A task with plant dynamics of $1 / \mathrm{s}^{2}$ is harder to control than a task with $1 / \mathrm{s}$ plant dynamics. The subjects trained to an average tracking error of 35 degrees. When the subjects enter the transfer phase the average tracking error remains in the vicinity of the trained asymptotic tracking error. The final average asymptotic tracking error is equal to 32.86 degrees. The average tracking error improved by 15.43 degrees over the total number of runs conducted. The difference between the asymptotic tracking for the training phase and transfer phase is equal to 2.14 degrees.

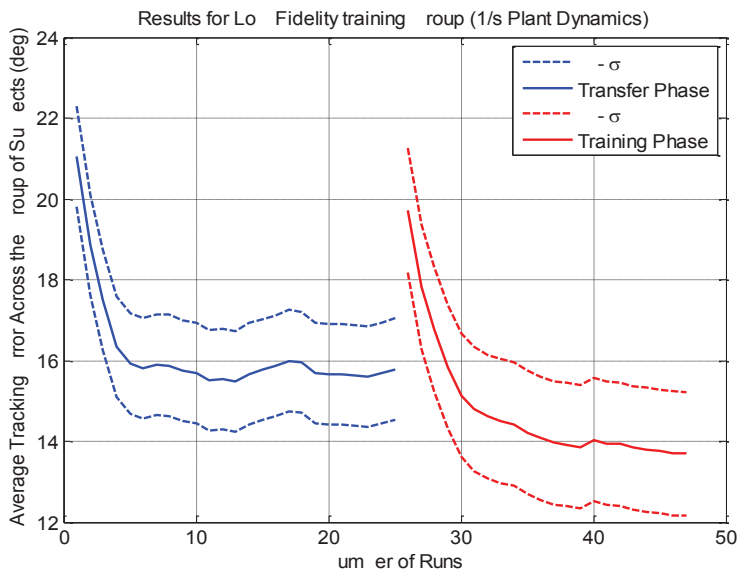

Figure 21 Results for Low Fidelity Training Group (1/s)

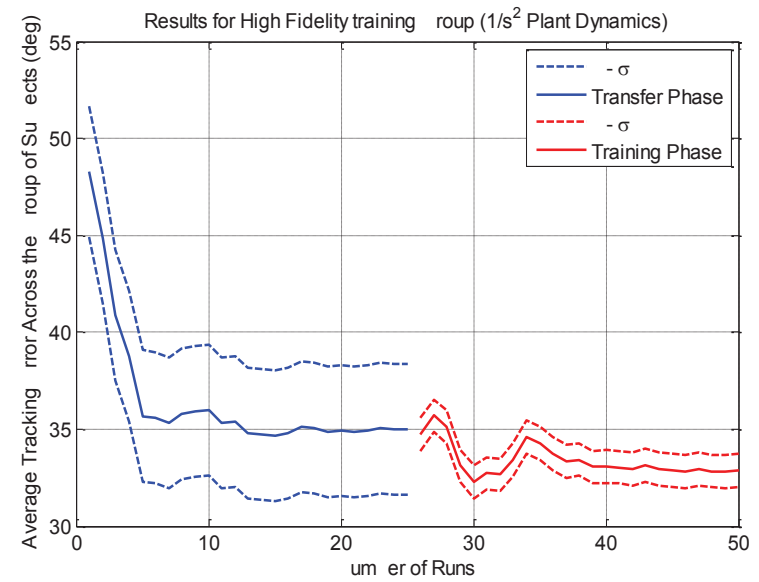

Figure 22 Results for High Fidelity Training Group $\left(1 / \mathrm{s}^{2}\right)$

Figure 23 shows the average tracking error between subjects for the low fidelity transfer group with $1 / \mathrm{s}^{2}$ plant dynamics. The low fidelity transfer group generally has a higher tracking error than the high fidelity training group throughout all the runs conducted. The tracking error for the low fidelity training group after they transferred did not drastically increase from the asymptotic performance obtained in training. The subjects were able to maintain their performance as they transferred to the high fidelity control loader simulation. This differs from the results for $1 / \mathrm{s}$ plant dynamics in which the tracking error drastically increases as the subjects transfer. For both plant dynamics, as the low fidelity training group transfers, they improve their tracking error further than the high fidelity training group when they transfer. The asymptotic tracking error for the training phase and transfer phase is equal to 41.55 degrees and 34.62 degrees respectively. The difference between the two asymptotic tracking errors is equal to 6.93 degrees.

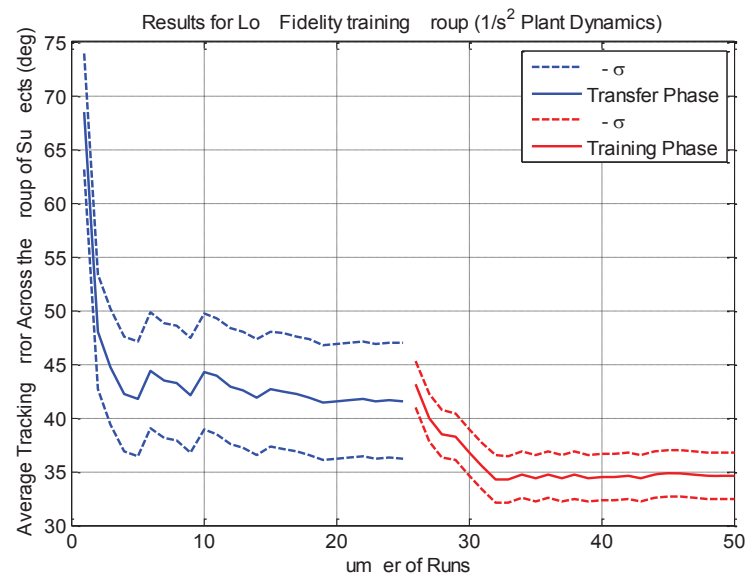

Figure 23 Results for Low Fidelity Training Group (1/s $\left.{ }^{2}\right)$ 
The results for both the high fidelity and low fidelity subject groups with $1 / \mathrm{s}$ and $1 / \mathrm{s}^{2}$ plant dynamics is shown in Figures 24 and 25 respectively.

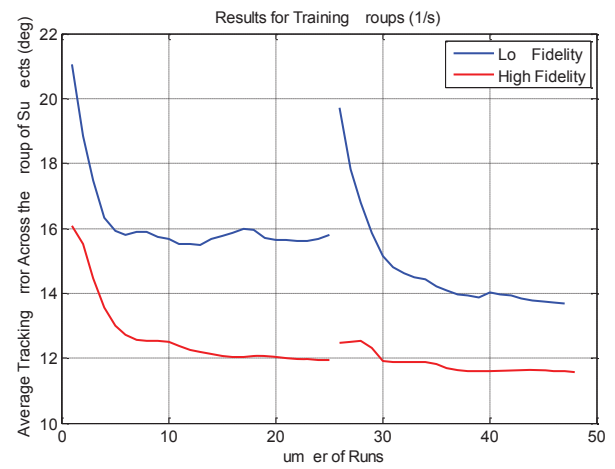

Figure 24 Results for High and Low Fidelity Training Groups (1/s)

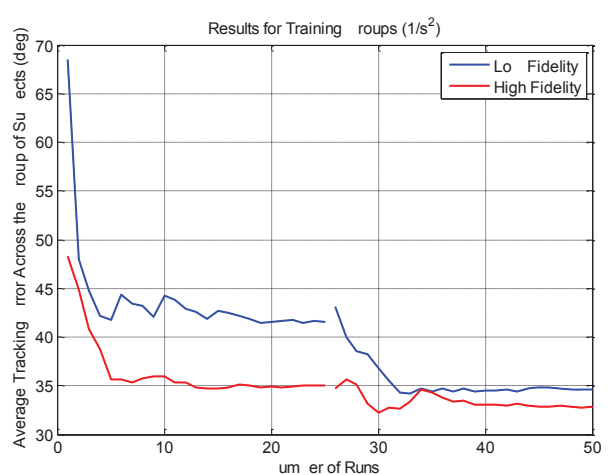

Figure 25 Results for High and Low Fidelity Training Groups $\left(1 / \mathrm{s}^{2}\right)$

In Figures 24 and 25, the high fidelity training group has a lower tracking error than the low fidelity training group. The difference between the final asymptotic tracking error performance for the high and low fidelity training groups is more substantial for $1 / \mathrm{s}$ than $1 / \mathrm{s}^{2}$ plant dynamics

\section{Summary and Conclusions}

It seems in general that the low fidelity group has poorer performance and that they do not transfer as well. Hence on the surface the hypothesis that there would be little difference between the two groups is not supported. The fact that we have not completed all the subjects or had sufficient time is due to the paper deadline. Perhaps once the complete set of subjects has been run the aggregate data might change. Although, observing the individual data there is no basis for that speculation. However it is our intention to determine statistical significance of the data. We also intend to perform Power Spectral Density analysis on the complete data set. In the past we have found that in human control research that the PSD indicates control behavior differences that are not revealed in tracking error performance.

It would appear that the low fidelity training subjects adapt better to the high fidelity control loader simulation for the harder task using $1 / \mathrm{s}^{2}$ plant dynamics. Plant dynamics of $1 / \mathrm{s}^{2}$ cause the pilot to compensate more leading to an excess in a commanded bank angle. Too much control inceptor deflection for $1 / \mathrm{s}^{2}$ plant dynamics can cause the output bank angle to grow in magnitude. When the low fidelity training group transfers the high fidelity control inceptor, the high force gradient could cause the subject to command less control inceptor deflection. Since the commanded input of the subject for $1 / \mathrm{s}^{2}$ plant dynamics is roll acceleration, too much control inceptor deflection can cause the bank angle to change rapidly which is difficult to null. For $1 / \mathrm{s}$ plant dynamics, the reason for increased tracking error initially could be that subjects cannot respond in a timely fashion to the disturbance since the high force gradient restricts the motion. $1 / \mathrm{s}$ plant dynamics are less sensitive than $1 / \mathrm{s}^{2}$ to control inceptor deflection.

In the future we have agreements to repeat this experiment at a number of laboratories in North America and in Europe. This series of experiments should provide a large data set for analysis. 


\section{References:}

Bailey, R.E. and L.H. Knotts, Interaction of Feel System and Flight Control System Dynamics on Lateral Flying Qualities, NASA Contractor Report 179445, NASA CR-179445, 1990

Gum, Don R., Modeling of the Human Force and Motion-Sensing Mechanisms, Air Force Human Resources Laboratory-Advanced Systems Division, AFHRL-TR-72-54, June 1973

Hess, Ronald A., "A Model-Based Theory for Analyzing Human Control Behavior," Advances in Man-Machine Systems Research, Volume 2, 1985, pp. 129-175

Hosman, Ruud J.A.W, David A. Abbink, and Frank M. Cardullo, "The Neuromuscular System,” AIAA Modeling and Simulation Technologies Conference, Toronto, Canada, August 2010

Johnston, Donald E. and Bimal L. Aponso, Design Considerations of Manipulator and Feel System Characteristics in

Roll Tracking, NASA Contractor Report 4111, NASA CR-4111, 1988

Levison, William H., A.M. Junker, "Effects of Simulator Delays on Performance and Learning in a Roll-Axis Tracking

Task," Fifteenth Annual Conference on Manual Control Wright State University, Dayton, Ohio, March 20-22, 1979

Magdaleno, R.E. and D.T. McRuer, Experimental Validation and Analytical Elaboration for Models of the Pilot's

Neuromuscular Subsystem in Tracking Tasks, NASA Contractor Report 1757, NASA CR-1757, April 1971

Mitchell, David G. and Bimal L. Aponso, Effects of Cockpit Lateral Stick Characteristics on Handling Qualities and

Pilot Dynamics, NASA Contractor Report 4443, June 1992

NASA.gov. (2010, May 16) THE FLIGHT SIMULATION FACILITIES Retrieved from

http://scap.hq.nasa.gov/docs/LaRC_Flight\%20Simulation.pdf.

Takats, Jim, "Flight Control System Simulation.” Flight \& Ground Vehicle Simulation Update, 2011 